Relations industrielles

Industrial Relations

\title{
Yves DELAMOTTE : Le droit du travail en pratique. Paris, Les Éditions d'organisation, 1983, 319 pp., ISBN 2-7081-0541-8
}

\section{Gérard Dion}

Volume 39, numéro 2, 1984

URI : https://id.erudit.org/iderudit/050044ar

DOI : https://doi.org/10.7202/050044ar

Aller au sommaire du numéro

Éditeur(s)

Département des relations industrielles de l'Université Laval

ISSN

0034-379X (imprimé)

1703-8138 (numérique)

Découvrir la revue

Citer ce compte rendu

Dion, G. (1984). Compte rendu de [Yves DELAMOTTE : Le droit du travail en pratique. Paris, Les Éditions d'organisation, 1983, 319 pp., ISBN 2-7081-0541-8]. Relations industrielles / Industrial Relations, 39(2), 402-403.

https://doi.org/10.7202/050044ar

Tous droits réservés (C) Département des relations industrielles de l'Université Laval, 1984
Ce document est protégé par la loi sur le droit d'auteur. L'utilisation des services d'Érudit (y compris la reproduction) est assujettie à sa politique d'utilisation que vous pouvez consulter en ligne.

https://apropos.erudit.org/fr/usagers/politique-dutilisation/ 
conceptuels apportés. Cette approche facilite les passages d'un concept au suivant: le lecteur constatera que beaucoup d'idées intéressantes y sont émises, tant pour le travail dans le secteur secondaire que dans le secteur tertiaire.

Le texte suivant, intitulé Technologie et emploi: un inventaire de stratégies nationales, décrit pour dix pays industrialisés - huit d'Europe, le Japon et les États-Unis - les stratégies et politiques nationales employées face à l'introduction de nouvelles technologies et aux conséquences de ces dernières sur l'emploi. Ce texte permet en même temps de visualiser le contexte économique et social propre à chacun de ces pays.

Finalement, Les impacts de l'informatisation dans les imprimeries du Québec rapporte les résultats d'une recherche effectuée au sein d'une industrie typiquement composée de PME. Encore une fois, cette étude suppose concrètement l'hypothèse selon laquelle il y a de nombreux points communs dans une même industrie, quant à la gestion de l'introduction de technologies. Cependant, les différences sont tout aussi remarquables et ne peuvent être négligées.

Globalement, ces huit textes fournissent une toile de fond intéressante quant aux caractéristiques des nouvelles technologies et à leur impact, tant au niveau de l'entreprise qu'au niveau national. Ces documents descriptifs sont orientés vers la pratique et sont facilement compréhensibles. L'un des avantages principaux réside dans le fait qu'il soit possible de lire n'importe lequel sans nécessairement avoir à lire tous les autres. Nul doute que ces textes peuvent sûrement aider et intéresser tout lecteur concerné par le virage technologique et par ses conséquences.

Jean NOLLET

Ecole des Hautes Etudes

Commerciales de Montréal
Le droit du travail en pratique, par Yves Delamotte, Paris, Les Éditions d'organisation, 1983, 319 pp., ISBN 2-7081-0541-8

Comme il fallait s'y attendre avec l'avènement d'un gouvernement socialiste au pouvoir, la législation du travail en France a connu des transformations profondes en 1982. Il ne s'agit pas seulement d'un rajeunissement mais d'une réforme marquant un tournant comme celles qui avaient été effectuées en $1864,1884,1936,1946$ et 1950. Un tiers du Code du travail a été abrogé et remplacé par des dispositions législatives nouvelles.

L'ouvrage du professeur Delamotte tient compte de ces développements et cherche à en dégager la portée. Il n'a pas été conçu à l'intention des étudiants en droit ni des juristes, mais plutôt pour ceux qui veulent acquérir des connaissances en droit du travail et particulièrement pour ceux qui, dans la pratique de leur vie professionnelle, ont besoin de connaître les règles qui régissent la situation des salariés et les rapports collectifs du travail, d'où le titre Le droit du travail en pratique.

Après une courte introduction historique, l'ouvrage est constitué de vingt-quatre chapitres classés en quatre parties. Suivent plusieurs annexes, des indications bibliographiques et enfin un index onomastique.

La première partie présente les organismes administratifs en matière de travail et d'emploi ainsi que les sources du droit. La seconde est consacrée à l'accès et à la perte de l'emploi ainsi qu'aux aléas de la vie professionnelle. Elle traite des différentes sortes de contrat de travail: à durée déterminée, travail temporaire, travail à temps partiel, de la résiliation du contrat de travail, du licenciement, de la cessation du contrat et enfin des prérogatives de la direction.

La troisième partie aborde le salaire et les conditions de travail: le salaire; participation et actionnariat; durée de travail et aménagement du taux de travail; jours fériés et congés; hygiène et sécurité; comité d'hygiène, de sécurité et des conditions de travail ainsi 
que l'expression des salariés et la médecine au travail; enfin, les différentes catégories de personnel dans l'entreprise.

La quatrième partie touche les relations collectives de travail: le syndicat; la convention collective; les délégués du personnel; le comité d'entreprise; le droit syndical dans l'entreprise; la protection des représentants du personnel contre le licenciement et le délit d'entrave au fonctionnement des institutions représentatives et l'exercice du droit syndical; enfin, les conflits du travail et la grève.

Le lecteur canadien, et particulièrement le lecteur québécois, sera intéressé à prendre connaissance de cet ouvrage pour diverses raisons.

Il constatera rapidement que, malgré que l'on déplore ici le juridisme dans les relations du travail et le développement accru de cette tendance en ces dernières années, ce n'est rien à côté de la multitude de dispositions législatives et réglementaires que l'on rencontre en France. Encore faut-il noter, comme le soulignait un article récent publié dans Relations industrielles, (vol. 38, no 1, 1983, pp. 23-24) qu'il est indispensable de faire la part des choses entre un texte de loi et son application, alors que prévaut souvent un pragmatisme accepté par les partenaires sociaux.

Ensuite, s'il est toujours utile de connaître ce qui se fait ailleurs, on doit se garder d'importer des mesures particulières sans tenir compte des principes et d'une cohérence qui marquent habituellement tout le régime juridique.

À partir cependant des mêmes principes comme le droit au travail et le droit d'association, en les transposant dans la législation, on peut en arriver à des dispositions totalement différentes. Ainsi, en ce qui regarde au Québec le contrôle exercé par le syndicat sur les salariés au cours d'une grève (mesures antibriseurs de grève). En France, «la grève est décidée par les salariés eux-mêmes, exerçant chacun le droit qui lui est reconnu par la Constitution. Certains salariés peuvent décider de ne pas faire la grève et le droit français préserve alors la liberté de travail. L'employeur est tenu, sauf force majeure, de leur fournir du travail. La grève n'apparaît donc pas, dans notre droit, comme résultant d'une décision des syndicats» (p. 292).

L'auteur présente son ouvrage comme un manuel. Il s'agit cependant d'un manuel d'un caractère particulier. Du manuel, il retient l'ordonnance, la concision, la clarté et la facilité de consultation. Mais il a le mérite d'être écrit dans un style vivant et un langage accessible, dépouillé de tout jargon pédant ou hermétique réservé aux initiés.

Gérard DION

Université Laval

In Search of Excellence: Lessons from America's Best-Run Companies, by T.J. Peters and R.H. Waterman Jr., New York, Harper and Row, 1982, XXVI350 pp., ISBN 0-06-015042-4

Peters et Waterman, dans cette étude, se penchent sur la source de l'excellence dans les meilleures entreprises états-uniennes, et concluent: "There is good news from America» (XXV). Ces bonnes nouvelles d'après ces auteurs sont:

«Good management practice today is not resident only in Japan. But, more important, the good new comes from treating people decently and asking them to shine, and from producing things that work. Scale efficiencies give way to small units with turned-on people. Precisely planned R. \& D. efforts aimed at big bang products are replaced by armies of dedicated champions. A numbing focus on cost gives way to an enhancing focus on quality. Hierarchy and three-piece suits give way to first names, shirts leeves, hoopla, and project-based flexibility. Working according to fat rule books is replaced by everyone's contributing.» (XXV) 